\title{
Global Maximum Power Point Tracking of PV Array Under Non- Uniform Irradiation Condition Using Adaptive Velocity Particle Swarm Optimization
}

\author{
Anang Tjahjono ${ }^{\mathrm{a}}$, Ainur Rofiq ${ }^{\mathrm{a}}$, Moh Hisyam Faiz ${ }^{\mathrm{a}}$, Dimas Okky Anggriawan ${ }^{\mathrm{a}}$ \\ ${ }^{a}$ Department of Electrical Engineering, Politeknik Elektronika Negeri Surabaya, Kampus ITS, Jl. Raya ITS, Surabaya, 60111, Indonesia \\ E-mail: anang.tj@pens.ac.id; rofiq@pens.ac.id; hisyamfaiz9@gmail.com; dimas@pens.ac.id
}

\begin{abstract}
Non-uniform irradiation condition (NUIC) is a condition of differences irradiation level received by each Photovoltaic (PV) on PV array. NUIC of PV array causes the emergence of several power peaks (consisting of several local peaks and one global peak) in the power-voltage (P-V) characteristic curve. This condition can cause several algorithms (hill-climbing / P\&O, IC) that are unable to reach the global peak as they are trapped at a local peak. This paper proposes an Adaptive Velocity Particle Swarm Optimization (AVPSO) algorithm to search the global peaks/Global Maximum Power Point (GMPP) of PV arrays under NUIC. The proposed algorithm is a modification of the PSO algorithm. AVPSO algorithm able to adjust its own weight factor values and cognitive acceleration coefficients depend on the distance of the particle's position now with the global best position during the tracking process. Adaptive weight factors can reduce the level of power or voltage oscillation during the tracking process until convergent, while the cognitive acceleration coefficient can prevent particles trapped at the local peak. Thus, the proposed AVPSO algorithm can reach GMPP with faster tracking time and low oscillation rates. In addition, this paper proposed an algorithm that can work both in static and dynamic NUIC patterns; thus, the proposed algorithm can track again when there is a change in global peak value in the PV array.
\end{abstract}

Keywords - NUIC; PV array; AVPSO; global peak; local peak; tracking time; convergent; oscillation.

\section{INTRODUCTION}

The increasing demand for electrical energy in the world is not proportional to the amount of fossil fuel reserved as the main fuel. The utilization of electric power generation with fossil fuel causes environmental degradation. Therefore, a lot of renewable energy is being developed. Photovoltaic (PV) is one of the most developed renewable energies. Solar energy is widely used due to its non-noise and non-pollution characteristics. Moreover, the photovoltaic system has various advantages, including low maintenance and long lifecycle.

Many researchers have concentrated on developing the PV generation. However, there is a challenge to get maximum PV power due to its non-linear characteristics, which change according to the environmental condition. In order to improve the efficiency of the photovoltaic power generation system, it is necessary to track the maximum power point of the photovoltaic system. There is a big challenge, especially in the case where PV arrays (more than one) are connected in series or parallel that work under non-uniform irradiation conditions (NUIC). Non-uniform irradiation condition (NUIC) is a condition of the difference irradiation levels received by PV arrays [1]. It can be caused by several factors such as dust that covers the surface of PV, shading from surrounding buildings, trees or poles, or other objects that can block the sun irradiation to the PV surface [1]. PV array under NUIC may drastically reduce the maximum power obtained [2]. Moreover, NUIC may cause internal heating of PV modules. To handle these issues, in the series connection of each PV module, a pass diode must be installed [3]. However, the intercalation of the bypass diode leads to another problem. The characteristics of PV array, which should only have one peak power, change to produce multiple power peaks (many local peaks and one global peak (GP) [4], as shown in Figure 5. Thus, there is a need for tracking this GP to ensure the maximum generation of power from the PV array under NUIC.

Several methods have been proposed to track power peaks of PV characteristics, which only have one peak. These methods include conventional methods such as, perturb and observe (P\&O) [6], [7] and incremental conductance (IC) [8]. There is a problem when conventional methods are applied under NUIC. This (conventional) method may not reach the global maximum power point (GMPP) because it is trapped at a low peak/local maximum power point (LMPP) [9]. 
Clearly, there is a need to overcome the multiple-peak characteristics with special MPPT technique that can track the GP (i.e., global maximum power point tracking or GMPPT). Several methods were developed to overcome multiple peak problem, such as particle swarm optimization (PSO) [10],[11], firefly algorithm (FA) [12], [13], Grey Wolf algorithm (GWA) [14], [15] etc. Although these methods were able to reach a global peak, it also caused new problems. PSO method incurs more PV voltage variations during the searching process and needs higher convergence time [16]. The firefly algorithm based on GMPPT is another technique to track GMPP with faster convergence, but it brings about severe PV voltage oscillations during searching. Grey wolf algorithm has a weakness, where it causes much larger variations in the PV array voltage during the tracking process and needs more time to converge [17]. To overcome the weaknesses of algorithms above, this paper proposes an Adaptive Velocity Particle Swarm Optimization (AVPSO); this method is adapted from the conventional PSO method, which is modified to overcome the issues encountered in other methods. AVPSO proposes an adaptive weight factor and adaptive cognitive acceleration coefficient to reduce the steady-state oscillations and to prevent the particles from getting trapped in local minima during the tracking process.

\section{MATERIALS AND METHOD}

The following sections provide several methods related to AVPSO. It also elaborates on all these methods before discussing APVSO in a separate section.

\section{A. Characteristics of PV Module at Normal Condition}

The characteristics are shown by the power versus voltage $(P-V)$ and the current versus voltage $(I-V)$. This curve informs the value of the power, current, and voltage that can be produced by the PV module at a certain irradiation level. Within the case of PV array that has series or parallel connection or combination of both, the characteristic of PV modules is an addition from the power produced by each PV module.

Figure 1 shows the characteristic of current versus voltage $(I-V)$. Figure 2 shows the difference of MPP from the same PV module with different irradiance levels. Figure 3, shown that when temperature level increases, the MPP of the same PV module decreases. Figure 1-3 represent a PV array with 3 PV module that arranged in series; each module's value is 50-watt peak. The parameter of the PV module is shown in Table 1.

TABLE I

PARAMETER OF EACH PV MODULE

\begin{tabular}{|l|l|}
\hline \multicolumn{1}{|c|}{ Parameter } & \multicolumn{1}{c|}{ Value } \\
\hline Maximum Power $\left(\mathrm{P}_{\max }\right)$ & $\mathbf{5 0 ~ W}$ \\
\hline Open Circuit Voltage $\left(\mathrm{V}_{\mathrm{oc}}\right)$ & $21.6 \mathrm{~V}$ \\
\hline Short Circuit Current $\left(\mathrm{V}_{\mathrm{sc}}\right)$ & $3.04 \mathrm{~A}$ \\
\hline Maximum Power Voltage $(\mathrm{VMP})$ & $17.6 \mathrm{~V}$ \\
\hline Maximum Power Current $(\mathrm{IMP})$ & 2.84 \\
\hline Test Condition & $1000 \mathrm{~W} / \mathrm{m}^{2}-25^{\circ} \mathrm{C}$ \\
\hline
\end{tabular}

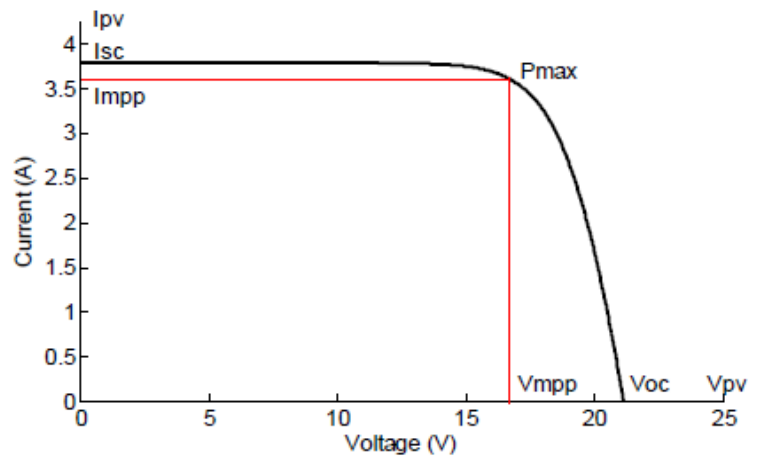

Fig. 1 I-V curve of PV Module

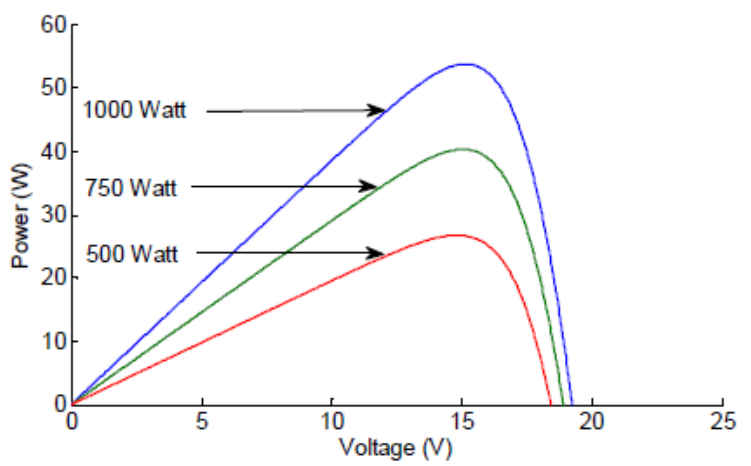

Fig. 2 P-V Curves at Several Irradiance Levels

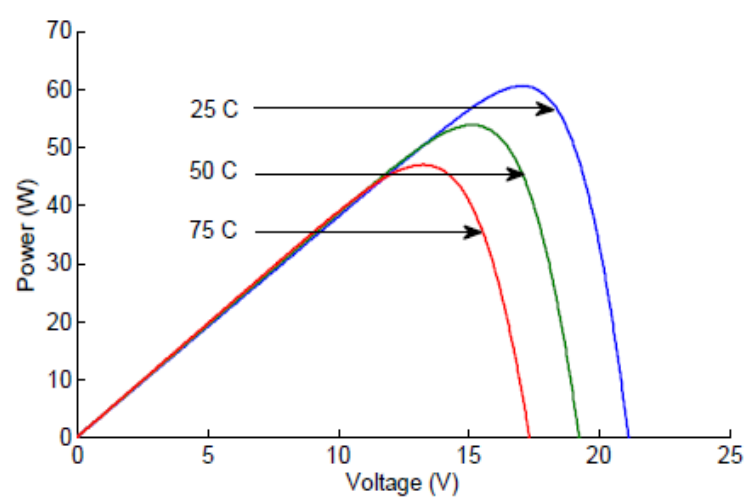

Fig. 3 P-V curves at Several Temperature Levels

\section{B. Equivalent Circuit of PV Modules}

A single diode composes the equivalent circuit of the PV module in parallel with a current source. The configuration of the PV module is shown in Figure 4. Only four components are used to describe the electrical characteristics of the PV module. A light generated current source is represented by $\mathrm{I}_{\mathrm{ph}}$. A single diode is connected in parallel with $\mathrm{I}_{\mathrm{ph}}$. The series and shunt resistances are represented by $R_{s}$ and $R_{s h}$.

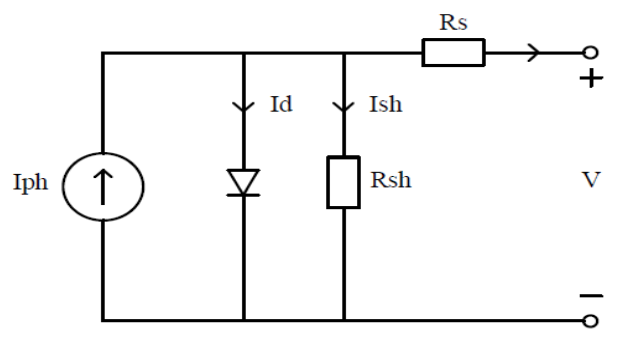

Fig. 4 Equivalent Circuit of PV Module 
Based on the equivalent circuit in Figure 4, the basic equation of the PV module is as follows:

$$
I=N_{p} \cdot I_{p h}-N_{p} \cdot I_{s} \cdot\left[\exp \frac{q\left(V+I_{R S}\right.}{N_{s} \cdot k \cdot T \cdot A}-1\right]
$$

$\mathrm{I}_{\mathrm{ph}}$ denotes the current generated by the PV module, $\mathrm{I}_{\mathrm{s}}$ denotes the saturation of diode current, $\mathrm{q}$ denotes the electron charge $(1.6 \times 10-19 \mathrm{C}), \mathrm{k}$ denotes the Boltzman constant $(1.38$ $\times 10-23 \mathrm{~J} / \mathrm{K}), \mathrm{T}$ denotes the PV cell temperature, A denotes the ideal factor, $R_{S h}$ is the shunt resistance, $R_{S}$ is the series resistance, $N_{s}$ is the number of PV cells in series. $N_{p}$ is a few $\mathrm{PV}$ cells in parallel.

\section{Coupled Inductor SEPIC Converter}

The DC-DC converter to track the MPP of the PV array is a SEPIC converter with a coupled inductor. This type of converter has high efficiency [18], and with a coupled-type of an inductor, it can decrease output current ripple [19]. The input current, voltage, and power continuously change due to varying irradiance and temperature conditions. Moreover, the duty cycle continuously changes to track the MPP of the PV module. The result of the proposed algorithm should be used to set the value of the duty cycle to track the MPP. Table 2 shows the parameters of the coupled inductor SEPIC converter.

TABLE II

PARAMETER OF SEPIC CONVERTER

\begin{tabular}{|l|l|}
\hline \multicolumn{1}{|c|}{ Parameter } & \multicolumn{1}{c|}{ Value } \\
\hline Inductor 1 & $602 \mathrm{uH}$ \\
\hline Inductor 2 & $565 \mathrm{uH}$ \\
\hline Capacitor 1 \& 2 & $731.5 \mathrm{uF}$ \\
\hline Resistor & $5.81 \Omega$ \\
\hline Switching frequency & $40 \mathrm{KHz}$ \\
\hline
\end{tabular}

\section{PV Array Under Non-Uniform Irradiation Condition} (NUIC)

PV array is composed of PV modules in series or parallel or combination of both to obtain sufficient power. The series connection can produce multiple voltages of the PV module, while a parallel connection can produce multiple currents with the same voltage. When all modules of PV array receive uniform radiation, the maximum power output of the PV array is equal to the sum of the maximum power all the modules [2]. However, when each module of PV array gets different irradiance levels, the power produced by the PV array becomes unstable, which is harmful to the normal operation of the PV array. This condition is called a nonuniform irradiation condition (NUIC).

Several factors can cause the differences between irradiance that receive each PV module. The dust that covers the surface of PV, shading from surrounding buildings, trees, or poles or other objects that can block the sun irradiation to the PV surface can cause different irradiation received by each PV module. To overcome the influence caused by NUIC and improve the efficiency of PV generation, it is necessary to study the impact on output characteristics of PV array under NUIC.

\section{1) PV Array Model Under NUIC}

In this case, the characteristic of the PV array taken from the same module like a reasonable condition above. Three modules PV 50WP arranged in series. PV array under NUIC may cause the maximum power obtained is drastically reduced [2]. Moreover, NUIC may cause internal heating of $\mathrm{PV}$ modules. To handle these issues, in series connection, each PV module must be installed bypass diode. Figure 5(a), shows the configuration of PV array under NUIC with bypass diode. Figure 5 (b) shows the $P-V$ characteristic of PV array under NUIC.

In the case of PV array under NUIC, for the seriesconnected configuration employing bypass diodes [Figure 5a] can cause $P$ - $V$ characteristic produce multiple peaks, with one of the peaks being global peak (GP) [Figure 5b]. The amounts of peaks that produced also depends on the number of configuration PV modules. The increasing number of arranged PV modules allows the emergence of other power peaks. Moreover, the number of peaks depends on the variation of irradiance level received by each PV module. For example, three PV modules connected in series, but only one of all modules that have different irradiance level, in case, $P$ $V$ characteristic of PV array only produce two power peaks.

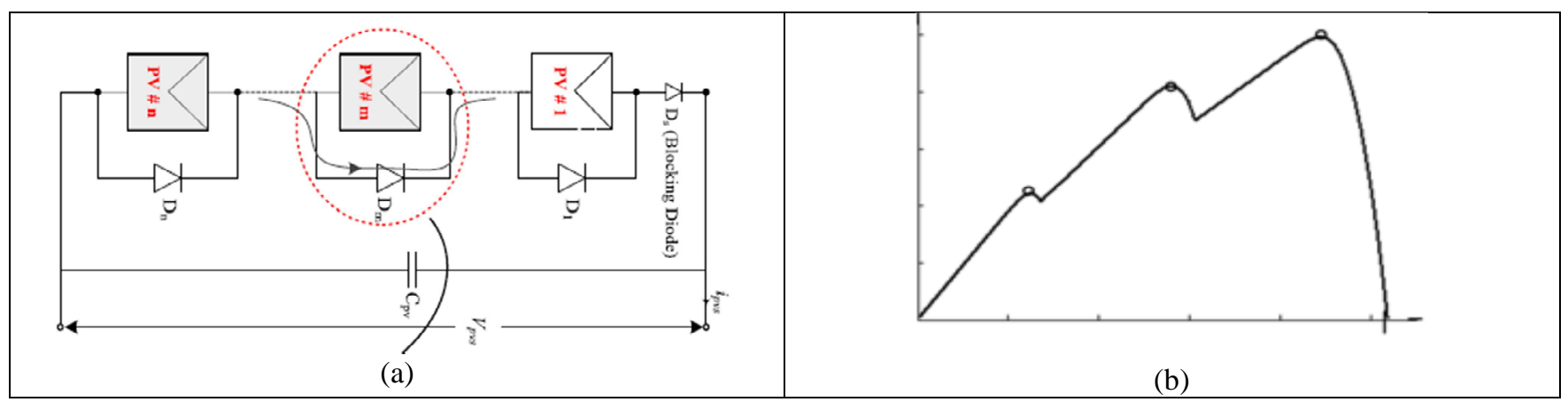

Fig. 5 (a) PV Array of Three PV Modules in a Series Configuration, (b) $P$-V Characteristic of PV Array

\section{2) Characteristic of PV (array) Under NUIC}

In the previous section, it was explained that the number of power peak produced depends on the number of arranged PV modules and the difference in irradiance values. Furthermore, the global peak (GP) position depends on the position of the PV module, which gets the highest level of irradiance. Table 3 below shows that several cases are carried out. Starting from uniform conditions, there is one difference in irradiation 
in PV modules, and all modules have different levels of irradiation. When each characteristic curve of each case is compared, there will be a noticeable difference in patterns.

TABLE III

THE DIFFERENCE IRRADIANCE LEVEL OF PV ARRAY

\begin{tabular}{|c|c|c|c|}
\hline \multirow{2}{*}{ PV array cases } & \multicolumn{3}{|c|}{ Radiation intensity $\left(\mathbf{W} / \mathbf{m}^{\mathbf{2}}\right)$} \\
\cline { 2 - 4 } & Module 1 & Module 2 & Module 3 \\
\hline 1 & 1000 & 1000 & 1000 \\
\hline 2 & 1000 & 1000 & 600 \\
\hline 3 & 400 & 1000 & 700 \\
\hline 4 & 400 & 600 & 1000 \\
\hline
\end{tabular}

Table 3 above indicates that case 1, which have uniform irradiance on each PV module, able to produce normal PV characteristic with only one peak. Case 2, where one PV module has different irradiance since PV array produces two peaks i.e., GP and LP with a lower value. In case 3, each PV module has different irradiance with the second module has the highest irradiance than all. In this case, the characteristic curve produces three peaks, i.e., one GP, and two LP. In this case, GP position in the middle of all peak that is due to the highest radiation found in the second module, which is in the middle of the PV array arrangement. Case 4, have a similar condition with case 3 , but in this case, the highest irradiance received by the third module. So that the GP position also changes according to the position of the module that receives the highest radiation. Thus, the conventional tracking algorithm (e.g., P\&O and IC) will fail to track GP position when PV array under case 2-4 (NUIC). The conventional algorithm will be stuck if it found a peak even though it's a local peak (LP).

\section{E. The Proposed Adaptive Velocity PSO Algorithm}

\section{1) Original PSO Algorithm}

Kennedy and Eberhart first developed the PSO algorithm in 1995. It was inspired by the social behavior of bird flocking. They have unique behavior when looking for food; they will spread and inform other birds if they find the best meal. In the optimization context, swarm (bird) assumed to have a certain size with every start position of particles randomly located in space. Every particle assumed to have two parameters, position, and velocity. Every particle moves in a certain space and considering the best position. Every particle sends information on the best position to other particles so that other particles can adjust the position and velocity of every particle based on the information received concerning the best position information. The position of a particle is varied according to the following equation (2):

$$
x_{i}^{k+1}=x_{i}^{k}+v_{i}^{k+1}
$$

Where $x_{i}{ }^{k}$ denotes the position of the $i$ th particle after $k$ th iteration. $x_{i}^{k+1}$ denotes the next particle position after the update or the position of a particle in the next iteration $(k+1) . v_{i}{ }^{k+1}$ denotes the value of velocity for $i$ th particle when $k+1$ iteration. The velocity value affects the speed and accuracy of the tracking process. The equation calculates velocity is shown in equation (3).

$$
\left(v_{i}^{k+1}\right)=w \cdot v_{i}^{k}+c_{1} r_{1}\left(x_{l b i}-x_{i}^{k}\right)+c_{2} r_{2}\left(x_{g b_{-} i}-x_{i}^{k}\right)(3)
$$

with $k=1,2,3, \ldots n$ as the number of iteration, $x_{i}^{\mathrm{k}}$ representing the position of each particle at each iteration. $\mathrm{X}_{l b}$ and $\mathrm{x}_{g b}$ are the personal best position of each particle and the global best position of the swarm. $\mathrm{C}_{1}$ and $\mathrm{C}_{2}$ are the cognitive coefficient acceleration and social coefficient acceleration. $\mathrm{r}_{1}$ and $r_{2}$ are random values lie in the range $0-1$. W is the inertia weight, which also has a range value of $0-1$. The velocity and position of each particle in the swarm are updated using equation (2) and (3).

\section{2) Modified PSO Algorithm}

Many researches and applications have successfully applied PSO optimization. Therefore, several researches of PSO algorithm are developed; one of them proposed to reduce the level of oscillation during the tracking process. The value of $\mathrm{C}_{1}$ and $\mathrm{C}_{2}$ are very influential on velocity calculation; if the value of $\mathrm{C}_{1}$ and $\mathrm{C}_{2}$ are too high, it can cause large oscillation during tracking proses. Thus, the modified PSO proposes the modification of $\mathrm{C}_{1}$ and $\mathrm{C}_{2}$ value to decrease the oscillation level during the tracking process. In this case, if an original PSO often uses 1 for the value of $\mathrm{C}_{1}$ and $\mathrm{C}_{2}$, then modified PSO changes the value become 0,5 . This value applies to all particles and every iteration update.

\section{3) Adaptive Velocity PSO Algorithm}

The conventional algorithm will fail to track GMPP of PV array. The PSO algorithm above is proposed to overcome the impact of NUIC. However, the original PSO algorithm is currently being developed. The purpose of developing PSO algorithm is to reduce or decrease the oscillation level during the tracking process and to shorten tracking time. Modified PSO algorithm offer to reduce oscillation level during the tracking process by reducing cognitive coefficient acceleration and social coefficient acceleration $\left(\mathrm{C}_{1}\right.$ and $\left.\mathrm{C}_{2}\right)$, but it can extend tracking time. To overcome this problem, this paper proposed an adaptive velocity PSO algorithm to improve the GMPP tracking time, avoids local peak trapping problem, and reduces the voltage oscillation level of PV array during the tracking process. The proposed AVPSO algorithm presented several modifications on the particle update process, including the process of velocity calculation. This section proposes a number of modified parameters based on the current position and the best position.

a) Adaptive Weight Factor: The value of the weight factor affects the velocity value update. Weight factor will keep the moving particle when it found a local peak, so it cannot be trapped in LP. But, the value of the weight factor that remains constant has a problem; it can cause sustained oscillation even all the particle was converged in GP point because the particle velocity has a non-zero value. In order to keep the weight factor function, and overcome the negative impact when all particle was convergence on GP, thus an adaptive weight factor was proposed. This paper proposes weight factor, which can change its value based on the distance of the particle from the GP. The weight of a particle is varied according to the following equation.

$$
w_{i}^{k}=\left|\frac{x_{g b}-x_{i}^{k}}{V_{o c}}\right|
$$


Where $x_{g b}$ is the global best position of all particle, $x_{i}{ }^{k}$ denotes the position of the $i$ th particle after $k$ th iteration, and $V_{o c}$ is the open circuit voltage value of PV array. Based from this equation, each particle can determine each weight value depend on the distance between the $i$ th particle position and the GBP. Thus, the weight factor of the particle is high when it is far from the GBP and causes each particle to avoid unnecessary oscillation when it is near to GP.Adaptive Acceleration Coefficient: the value of the social and cognitive acceleration coefficient also determine the velocity value update process. Cognitive acceleration Coefficient $\left(\mathrm{C}_{1}\right)$ keeps the particle moving before it found GP. $\mathrm{C}_{1}$ and $\mathrm{r}_{1}$ are the values that prevent the particle from being trapped in LP. In the original PSO, $\mathrm{C}_{1}$ is selected by the programmer. This condition causes an increase within the tracking time because the value of $\mathrm{C}_{1}$ is not zero when it reaches the GP so that the particle makes an unnecessary movement.

This paper proposed the $C_{1}$ that can change its value based on its particle position from the global best position. Initially, when particles are far from the GP, there may be multiple minima located in between. Thus, the value of $C_{l}$ must be kept low to avoid particles from trapping in the local minima. When the particle has blessed to the GP, the local peak already passed, so the value of $C_{l}$ can be gradually increased to reduce convergence time. Thus, the adaptive value of $C_{l}$ is varied according to the following equation:

$$
C_{1}{ }^{k}=0.5\left(1-\left|\frac{x_{g b}-x_{i}{ }^{k}}{V_{o c}}\right|\right)
$$

The constant 0.5 is to limit the maximum value that can be achieved by $C_{1}$ to be 0,5 . This ensures the $C_{1}$ value is always less than $C_{2}$ or at most, is equal to $C_{2}$. Thus, the cognitive factor is never able to dominate over the social factor and pull the particle farther from the GP, especially for cases when the particle lies in a local minimum.

Original PSO also proposed a fixed value of Social Acceleration Coefficient $\left(\mathrm{C}_{2}\right)$, but it needs to be able to avoid the minimum local trapping and reduce the tracking time based on its condition/position. $\mathrm{C}_{2}$ almost similar to Cognitive acceleration Coefficient $\left(C_{1}\right)$; the $C_{2}$ value is presented for calculating the value by its self. To make the algorithm free from tuning requirements, the following formula for the value of $\mathrm{C}_{2}$ is proposed:

$$
C_{2}^{k}=\left(1+\left|\frac{x_{g b}-x_{i}^{k}}{V_{o c}}\right|\right)
$$

The formula presented above allows $C_{2}$ value to have 1-2 range values. It ensures that the value of $\mathrm{C}_{2}$ is higher than the initial stages when the particle was far from GP. The proposed formula made the particle moves quickly at the beginning of the process and can reduce unnecessary movement, so it can reduce tracking time. In this paper, $r_{1}$ and $r_{2}$ must be removed, because they can interfere with the calculation of $\mathrm{C}_{1}$ and $\mathrm{C}_{2}$.

b) Limits on Particle Velocity: The adaptive velocity equation that bases on adaptive weight factor and adaptive cognitive factor is as follows.

$\left(v_{i}^{k+1}\right)=\left|\frac{x_{g b}-x_{i}^{k}}{V_{o c}}\right| \cdot v_{i}{ }^{k}$

$$
\begin{aligned}
& +\left\{0.5\left(1-\left|\frac{x_{g b}-x_{i}^{k}}{V_{o c}}\right|\right)\right\}\left(x_{l b i}-x_{i}^{k}\right) \\
& +\left\{\left(1+\left|\frac{x_{g b}-x_{i}^{k}}{V_{o c}}\right|\right)\right\}\left(x_{g b_{-} i}-x_{i}^{k}\right)
\end{aligned}
$$

equate all denominators by multiplying all summations with $\mathrm{v}_{\mathrm{oc}}$ values, so the simpler equation becomes as follows.

$$
\begin{aligned}
& \left(v_{i}{ }^{k+1}\right)=\left|x_{g b}-x_{i}{ }^{k}\right| \cdot v_{i}{ }^{k} \\
& +\left\{0.5 *\left(1-\left|x_{g b}-x_{i}{ }^{k}\right|\right)\right\}\left(x_{l b i}-x_{i}{ }^{k}\right) \\
& +\left(1+\left|x_{g b}-x_{i}{ }^{k}\right|\right)\left(x_{g b_{-} i}-x_{i}{ }^{k}\right)
\end{aligned}
$$

In case under NUIC has multiple peaks, the distance between particle is so far, especially at the beginning process. The update velocity value may reach large value; this condition may cause a problem. The particle may skip some intermediate peaks, and maybe which one is GP. This will lead to erroneous tracking of the GMPP. Therefore, to ensure that none of the peaks are passed during the tracking process, the update velocity value must be limited to a maximum value as the following equation.

$$
\begin{gathered}
0<\left|v_{i}{ }^{k+1}\right|<V_{\text {max }} \\
V_{\text {max }}=\left(0.8 * V_{o c}\right) / \mathrm{N}
\end{gathered}
$$

Equation (8) shown that the velocity value less than $\mathrm{V}_{\max }$. $\mathrm{V}_{\max }$ of PV array may be calculated from equation (10). Voc denotes the open-circuit voltage, and $\mathrm{N}$ denotes the number of PV modules connected in the PV array. The proposed limiting value ensures that none of the peaks are skipped while tracking the process of GMPP.

\section{4) Implementation of The Proposed AVPSO Algorithm}

This section will describe the implementation of the proposed algorithm and the validation of the advantages it offered. Figure 6 shows block diagram of the prototype based MPPT. Meanwhile, the AVPSO algorithm to overcome the impact of PV array under NUIC is presented in the flowchart in fig 7. Here, SEPIC converter serves as interface and actuator of MPPT between PV array and load. The proposed algorithm controls the DC-DC SEPIC converter operates at the optimum duty cycle corresponding to GMPP.

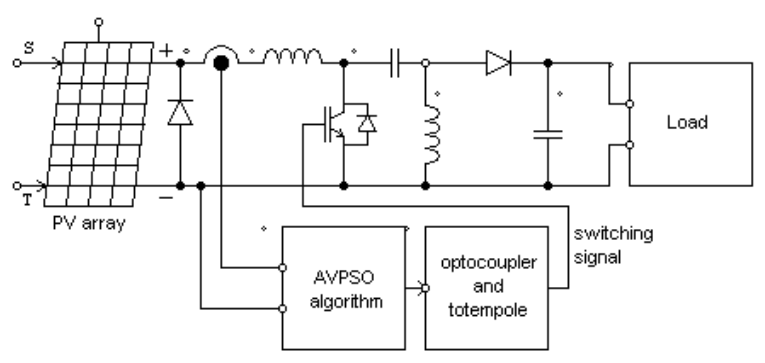

Fig. 6 Block Diagram of the prototype Based MPPT Scheme

The steps of the proposed algorithm toward MPPT are described as follows:

1) Step 1: initial parameter of the AVPSO algorithm. Initially, to determine the number of particles to be spread. The particle's position initially may be random or specified by the programmer. The initial positioning of the particle will 
affect the tracking process. In this paper, the particle is a duty cycle that will be given to the SEPIC converter. Further, it will determine how many particles update their positions; in this paper, each particle can update their position until ten iterations.

2) Step 2: fitness evaluation, each particle has a different value; each particle will produce different voltage and current readings from the PV array. Voltage and current value must be calculated to determine how much power produced from each particle.

3) Step 3: Gbest and Pbest update process. If the power value for duty (i) is better than the best power in the history of duty (i), then set the current value as the new Pbest (i) value. After that, choose duty with the best power between the five duty cycles to be used as the Gbest value.

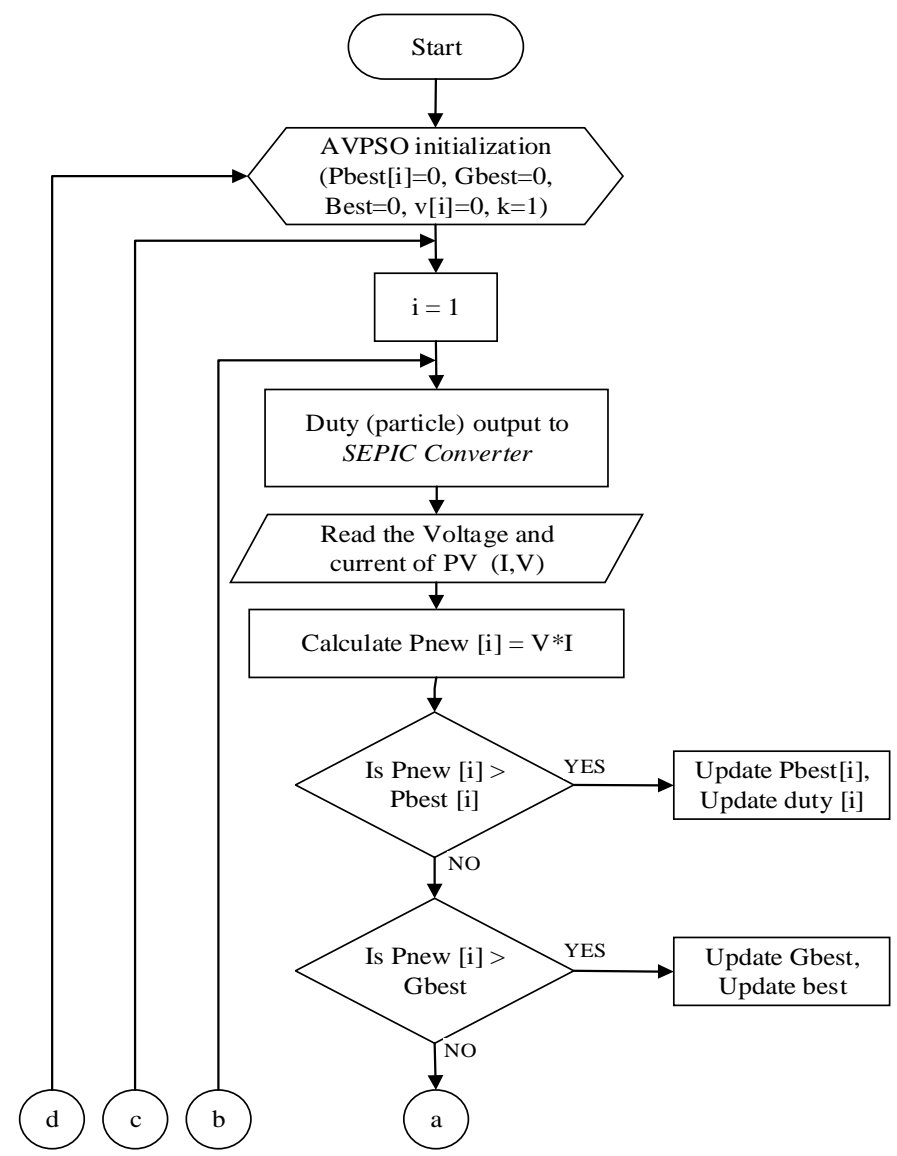

Fig. 7 Flowchart of the Proposed AVPSO Algorithm

\section{RESULTS AND DISCUSSION}

\section{A. Validation of The Proposed AVPSO Algorithm by Simulation}

To evaluate and analyze the performance of the proposed algorithm, PSIM model is developed to simulation studies. The block diagram to track the MPP is shown in Figure 6. The simulation parameters of the MPP tracker circuit (SEPIC

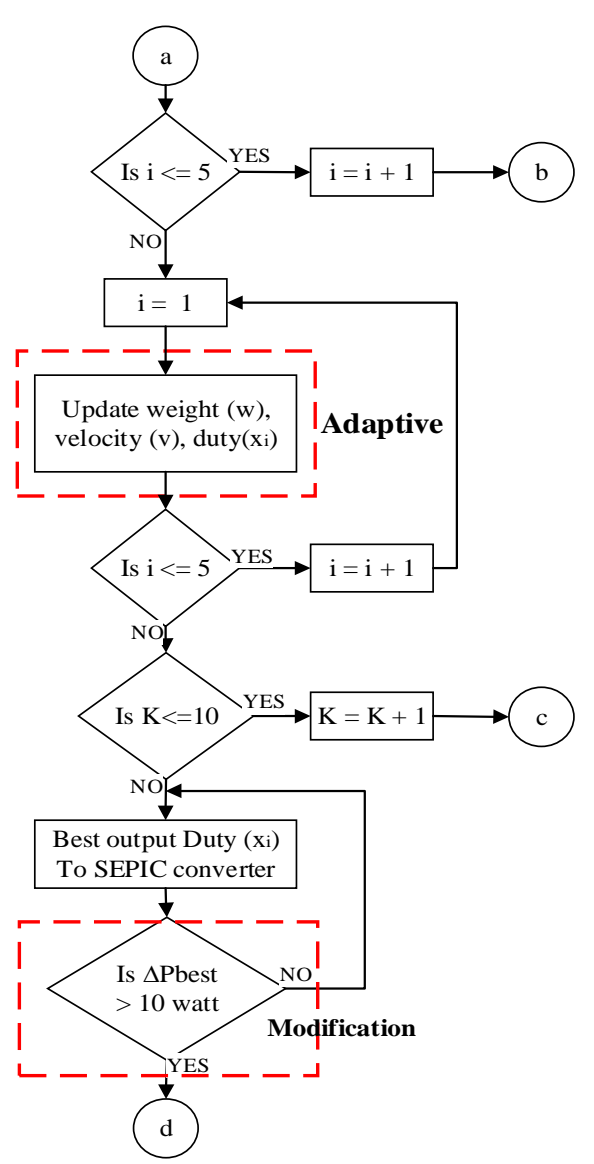

4) Step 4: velocity update and particle position update process. After all, particles have been evaluated, the velocity and particle position herd must be updated. In the AVPSO method, updates are made using the equations described in the previous section.

5) Step 5: convergent condition. The state of convergence is reached if the iteration value has reached the maximum number of iterations. The AVPSO algorithm will stop, and the best particle cycle in the iteration history will be used at the DC-DC converter. Convergence also may be achieved before the iteration runs out, but on the condition that all particles are at a GP.

6) Step 6: re-initialization. This step will be executed when a power change occurs when all the particles have converged. This paper proposed re-initialization when there is a change in a power greater than 10 watts. This step requires the algorithm to process from the beginning. converter) For the implementation of the proposed AVPSO algorithm are given in Table 4.

To validate whether the AVPSO algorithm can work, the power tracking result will be compared with the maximum power generated by the PV array. To find out the advantages of the proposed algorithm, the waves during the tracking process until the convergent point will be compared between the original PSO and AVPSO. The simulation result is shown in fig 8 . 
TABLE IV

SIMULATION PARAMETER OF MPPT WITH SEPIC CONVERTER

\begin{tabular}{|c|c|c|}
\hline No & Parameter & Specification \\
\hline 1 & PV modules & $\begin{array}{r}\text { Pmpp }=150 \mathrm{~W} ; \mathrm{Vmpp}=52.8 \mathrm{~V} \\
\text { Isc }=3.04 \mathrm{~A} ; \mathrm{Voc}=64.2 \mathrm{~V} \\
\mathrm{~T}=25^{\circ} \mathrm{C}\end{array}$ \\
\hline 2 & Inductor 1 & $602 \mathrm{uH}$ \\
\hline 3 & Inductor 2 & $565 \mathrm{uH}$ \\
\hline 4 & Capacitor $1 \& 2$ & $731.5 \mathrm{uF}$ \\
\hline 5 & resistor & $5.81 \Omega$ \\
\hline 6 & Switching Frequency & $40 \mathrm{KHz}$ \\
\hline
\end{tabular}

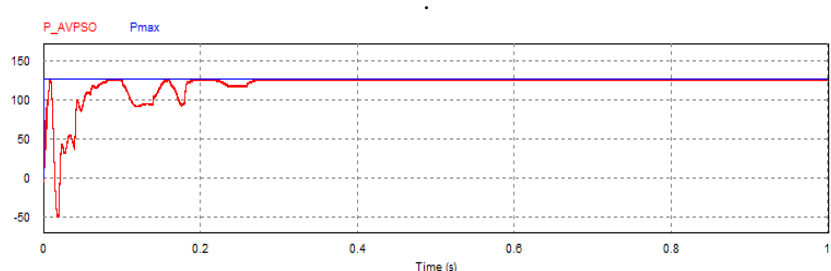

(a)

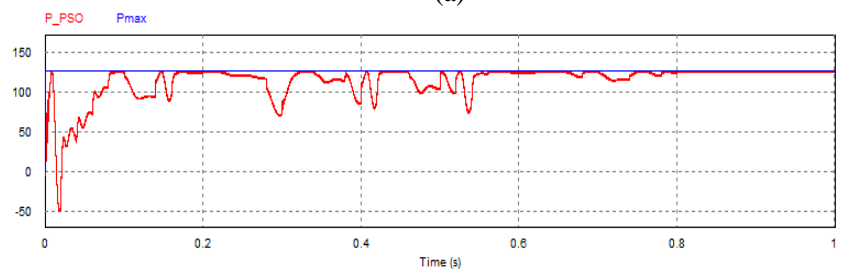

(b)

Fig. 8 Power Waveform (a) the AVPSO Algorithm, (b) Original PSO

From the two images above, the power resulted from the tracking process coincides with the maximum power (nominal) produced by the solar panel configuration. However, there are some differences. Based on Figure 8, AVPSO can reach a convergent point faster than the original PSO. Moreover, the original PSO performs unnecessary movement when it was approaching the convergent point, so it can extend the tracking time.

\section{B. Validation of The Proposed AVPSO Algorithm by Hardware Experiments}

To validate the real performance of proposed algorithm, this paper proposes implementation AVPSO using DC-DC SEPIC Converter and STM32F7 as a controller. Hardware experiment configuration can be seen in Figure 9, where the AVPSO algorithm is applied in the controller (STM32F7). Voltage and current, which is used to calculate the PV power, is obtained from the voltage sensor and current sensor, which is installed after the PV array. Both of them are the parameters used in the AVPSO algorithm. AVPSO technique to change the duty ratio to generate the control signal through the driver circuit to control the switching mosfet of SEPIC Converter.

1) Hardware experiment on normal condition (uniform irradiation): Hardware experiments have been carried on several conditions to validate AVPSO can work in normal condition (uniform irradiation) and can work under NUIC in various irradiation, and various combinations of PV array. The first three experiments have been carried on normal conditions; each PV module gets the same irradiation, high, medium, and low irradiation. This experiment aims to find out whether or not AVPSO also can work in normal conditions (uniform irradiation). Figure 9 shown the hardware experiment configuration using a resistor as a load.

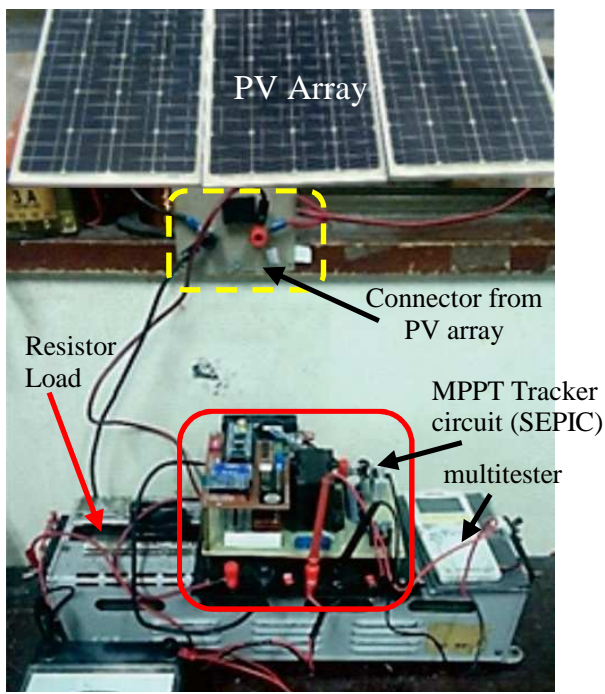

Fig. 9 Hardware Experiment Configuration

The experiment was carried on three different values of irradiation, $576 \mathrm{~W} / \mathrm{m}^{2}$ as low irradiation, $837 \mathrm{~W} / \mathrm{m}^{2}$ as normal irradiation, and $1072 \mathrm{~W} / \mathrm{m}^{2}$ at high irradiation. Each method was tested in each condition. Each method was tested in contiguous time to prove that there will not be any significant radiation change. The result of this experiment is shown in Table 5.

TABLE $V$

EXPERIMENTAL RESULT ON NORMAL CONDITION (UNIFORM IRRADIATION)

\begin{tabular}{|c|c|c|c|c|c|}
\hline $\begin{array}{c}\text { Irradiation } \\
\left(\mathrm{W} / \mathbf{m}^{2}\right)\end{array}$ & Method & $\begin{array}{c}\text { Pmax } \\
\text { (W) }\end{array}$ & $\begin{array}{c}\mathbf{P}_{\mathrm{MPPT}} \\
(\mathbf{W})\end{array}$ & $\begin{array}{c}\text { Acuracy } \\
(\%)\end{array}$ & $\begin{array}{l}\text { Tracking } \\
\text { Time (s) }\end{array}$ \\
\hline \multirow{3}{*}{576} & $\mathrm{P} \& \mathrm{O}$ & \multirow{3}{*}{61.78} & 56.24 & 91.03 & 7.7 \\
\hline & PSO & & 58.68 & 94.98 & 11.6 \\
\hline & AVPSO & & 61.36 & 99.32 & 6.03 \\
\hline \multirow{3}{*}{837} & $\mathrm{P} \& \mathrm{O}$ & \multirow{3}{*}{104.25} & 100.44 & 96.34 & 9.6 \\
\hline & PSO & & 100.45 & 96.35 & 6.7 \\
\hline & AVPSO & & 101.7 & 96.94 & 5.7 \\
\hline \multirow{3}{*}{1072} & $\mathrm{P} \& \mathrm{O}$ & \multirow{3}{*}{125.18} & 123.1 & 98.33 & 12.3 \\
\hline & PSO & & 123.24 & 98.45 & 6.1 \\
\hline & AVPSO & & 123.53 & 98.25 & 5.4 \\
\hline
\end{tabular}

$\mathrm{P}_{\mathrm{MAX}}(\mathrm{W})$ is a peak power from the PV characteristic curve at that time. $\mathrm{P}_{\mathrm{MPPT}}$ is a peak power (convergence condition) that can be reached by each method at that time. Accuracy is a power ratio that can be reached by each method compared with $\mathrm{P}_{\text {MAX }}$. Tracking time is how long each method reaches a convergence condition. In addition, Figure 10 shown the experiment result graph each method in 3 irradiation value.

Based on the result on Table 5, AVPSO is able to work on normal condition, it was proven from the peak power generated by AVPSO can approach the peak power from the characteristic curve. AVPSO have an average accuracy up to $98 \%$. Based on Figure 10, AVPSO can reach convergence condition faster than PSO and $\mathrm{P \& O}$ at all irradiation value. AVPSO have average tracking time less than 6 seconds, with lower oscillation level. 


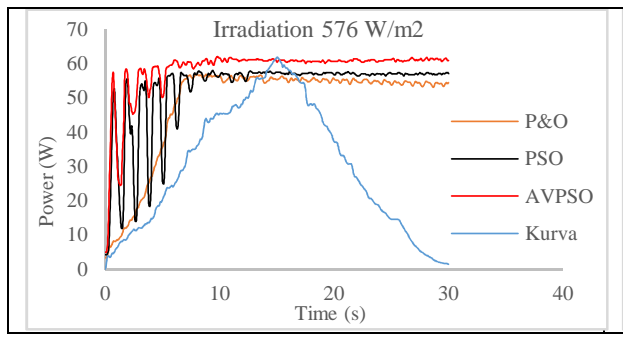

(a)

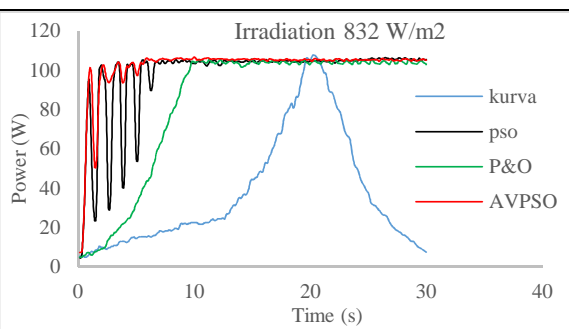

(b)

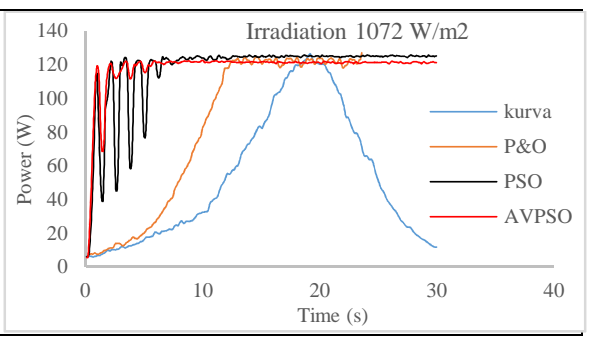

(c)

Fig. 10 Experimental Result of Each Method; a) at low irradiation; b) at medium irradiation; c) at high irradiation

Based on Figure10, it appears that the PSO standard has high-level oscillation until it reaches convergence condition. However, this problem was resolved by AVPSO. Based on Figure10, AVPSO just has a big oscillation in the first cycle. After that, it will gradually decrease until it reaches a convergence condition. This condition can simplify the process of voltage control in the output side. In this experiment, the ability of AVPSO to avoid local peak trapping is yet seen, because on normal condition (uniform radiation) PV array just generates one peak power, so that conventional method $(\mathrm{P} \& \mathrm{O})$ can approach the peak power.

2) Hardware Experiment Under NUIC: Hardware experiment under NUIC (Non-Uniform Irradiation Condition) was carried out to validate that AVPSO is able to overcome the weakness of the other methods. This experiment was also carried out under three different conditions to validate the reliability of the proposed algorithm. Non-Uniform Irradiation Condition made by partially covering the surface of the PV array. The different condition was made by change the amount and surface area of the closed PV.

The difference between the three combinations of PV surface closure appears in figure 11. In the first test, only part of one PV's surface was closed, and then two PV modules partially closed, and the last two PV modules partially closed but with a different position and area. Each combination will produce different characteristic curves. Each combination has a different peak value, peak position, and has a different amount of peak power; it all depends on the number of PV modules that have irradiation differences.

To validate that the proposed algorithm is better than several other algorithms, results comparison was carried out. The result of each method is compared against the characteristic curve generated by the PV array at this condition. This paper focuses on comparing the result of the tracking process, especially on oscillation level and tracking time until they reach convergent conditions and remain concerned about accuracy that related to the ability of the proposed algorithm to avoid local peak trapping.

Table 6 shown the experimental result of each method on each pattern. In this table, several pieces of information were shown. $\mathrm{P}_{\text {MAX }}(\mathrm{W})$ is a peak power from the PV characteristic curve at that time. $\mathrm{P}_{\mathrm{MPPT}}$ is a peak power (convergence condition) that can be reached each method at that time. Accuracy is a power ratio that can be reached by each method compared with $\mathrm{P}_{\mathrm{MAX}}$. Tracking time is how long each method reaches convergence condition. Oscillation is how much the graph oscillates (1 period) until it reaches convergence condition. Oscillation is related to tracking time and voltage variation during tracking process and it is very influential in the process of regulating output side.

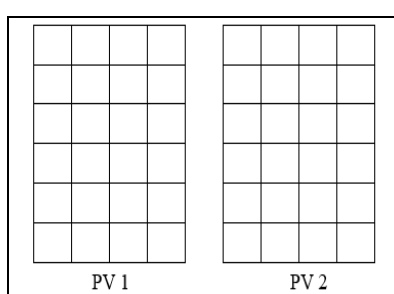

(a)

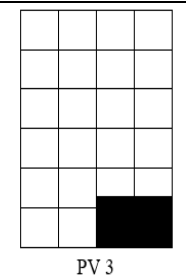

PV 3

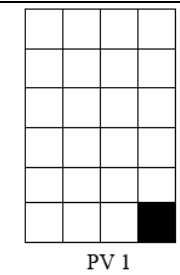

PV 1

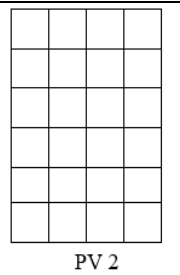

(b)

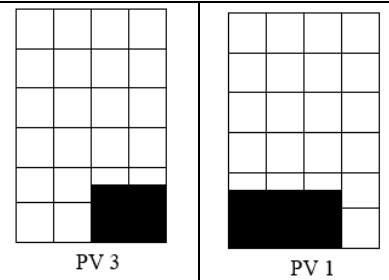

PV

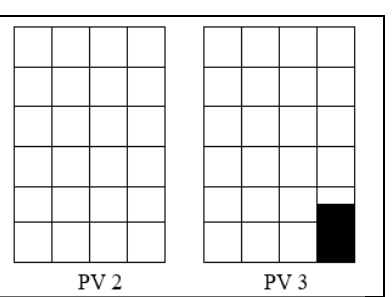

(c)

Fig. 11 Combination of PV Surface Closure (a) First Pattern; (b) Second Pattern; (c) Third Pattern

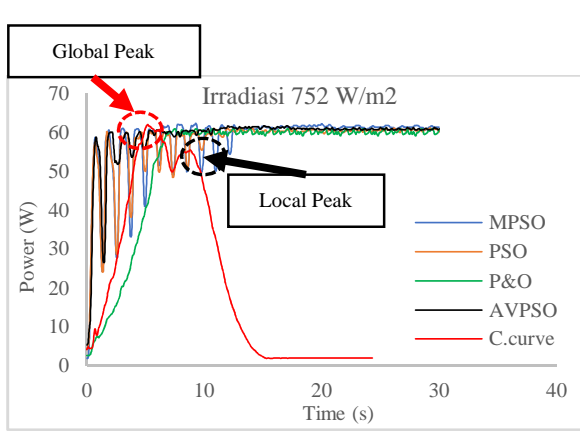

(a)

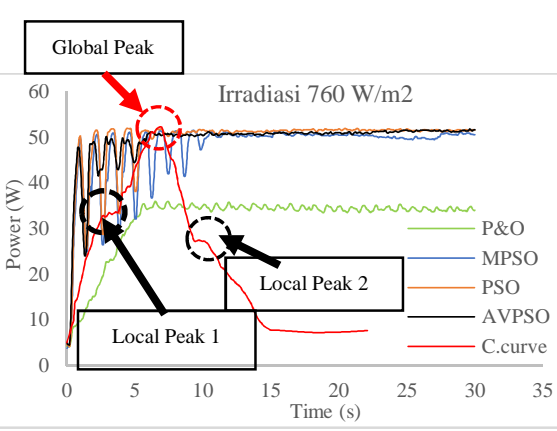

(b)

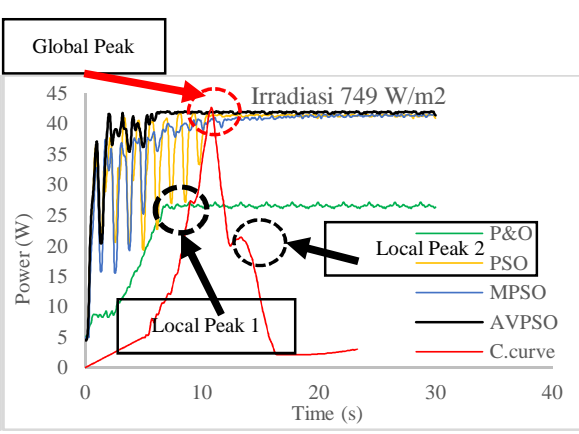

(c)

Fig. 12 Comparison the Experimental Result of each MPPT Method Under NUIC(a) the result at pattern 1;(b) the result at pattern 2;(c) the result at pattern 3 
TABLE VI

EXPERIMENTAL RESULT OF EACH METHOD UNDER NUIC

\begin{tabular}{|c|c|c|c|c|c|c|}
\hline Pattern & Method & $\operatorname{Pmax}(W)$ & $\mathbf{P}_{\text {MPPT }}(\mathbf{W})$ & $\begin{array}{c}\text { Accuracy } \\
(\%)\end{array}$ & $\begin{array}{c}\text { Tracking } \\
\text { time (s) }\end{array}$ & oscillation \\
\hline \multirow{4}{*}{1} & $\mathrm{P} \& \mathrm{O}$ & \multirow{4}{*}{62.04} & 61.19 & 98.6 & 6.8 & - \\
\hline & PSO & & 60.69 & 97.82 & 10.8 & 8 pulse \\
\hline & MPSO & & 61.74 & 99.51 & 12.5 & 10 pulse \\
\hline & AVPSO & & 61.81 & 99.62 & 5.9 & 5 pulse \\
\hline \multirow{4}{*}{2} & $\mathrm{P} \& \mathrm{O}$ & \multirow{4}{*}{52.29} & 35.58 & 68.04 & 5.6 & - \\
\hline & PSO & & 51.13 & 97.78 & 6.6 & 6 pulse \\
\hline & MPSO & & 50.84 & 97.22 & 11.4 & 8 pulse \\
\hline & AVPSO & & 50.95 & 97.43 & 6.2 & 4 pulse \\
\hline \multirow{4}{*}{3} & $\mathrm{P} \& \mathrm{O}$ & \multirow{4}{*}{42.73} & 26.98 & 63.14 & 6.7 & - \\
\hline & PSO & & 40.57 & 94.94 & 10.4 & 7 pulse \\
\hline & MPSO & & 41.89 & 98.03 & 11.9 & 9 pulse \\
\hline & AVPSO & & 41.91 & 98.08 & 6.1 & 5 pulse \\
\hline
\end{tabular}

Based on Figure12, the PV array under NUIC generates different characteristic curves according to their pattern. In Figure12 (a) (pattern 1), only two peaks power (one local peak and one global peak) are generated by PV array; there are two different irradiation values from $3 \mathrm{PV}$ arranged in series. On this pattern, a conventional algorithm $(\mathrm{P} \& \mathrm{O})$ is not trapped on the local peak because it through global peak first, then local peak; thus, it tried to maintain its position at the peak that was discovered first.

Conventional PSO also can reach global power for 10.8 seconds with eight pulses oscillation. This experiment is compared against the MPSO (modified PSO); it is a conventional PSO that has changed the value of cognitive coefficient acceleration and social coefficient acceleration $\left(\mathrm{C}_{1}\right.$ and $\left.\mathrm{C}_{2}\right)$. The value of $\mathrm{C}_{1}$ and $\mathrm{C}_{2}$ were changed to 0 from 1 (normal value). Based on the experimental result on Figure13, the proposed AVPSO can reach the global power peak (GPP) without being trapped in a local peak; it has short tracking time than the other method and also has a lower oscillation level.

Different characteristic curve was generated at pattern 2 (Figure12 b). It has 3 peak power, which consists of 2 local peaks and 1 global peak. Three power was generated because at this pattern have three different irradiation received by PV array, each PV module have different irradiation because it has differences in surface area that exposed to sunlight. Based on fig 15. (b) and Table 6, conventional algorithm $(\mathrm{P} \& \mathrm{O})$ was trapped on local peak 1 , because the nature of $\mathrm{P} \& \mathrm{O}$ that maintains its position at the first found peak. The proposed AVPSO can reach the global peak without trapping on a local peak. It has a shorter tracking time than the other methods and also has a lower oscillation level. For more details, the comparison of oscillation levels is shown in Figure 13.

The experimental result shown in Figure15 is the result of experiments carried out at normal conditions (uniform irradiation). The different experimental results of several methods are shown in the figure above (fig 14). The advantages of the proposed algorithm are shown, especially about the oscillation level. Conventional PSO and modified PSO has a stable decrease in oscillation until it converges, the value of cognitive coefficient acceleration causes it, and social coefficient acceleration $\left(\mathrm{C}_{1} \& \mathrm{C}_{2}\right)$ is constant/not changeable. Therefore, the adaptive value of weight factor and adaptive value of $\mathrm{C}_{1} \& \mathrm{C}_{2}$ on AVPSO able to overcome the problem.

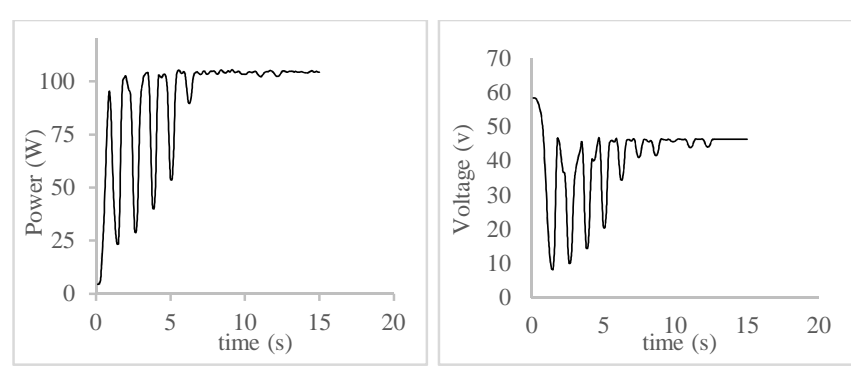

(a)

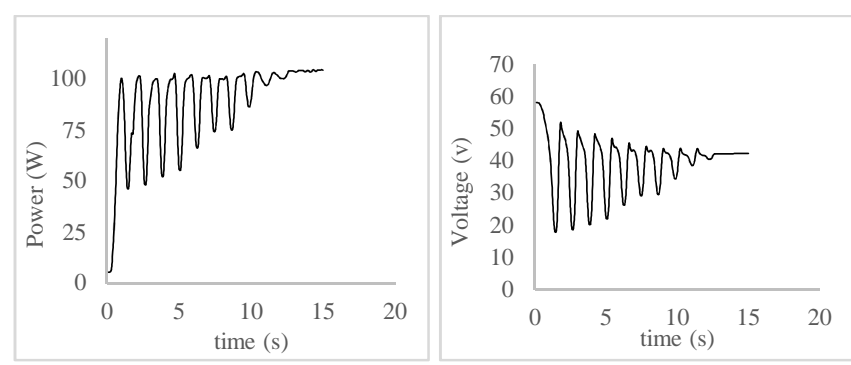

(b)

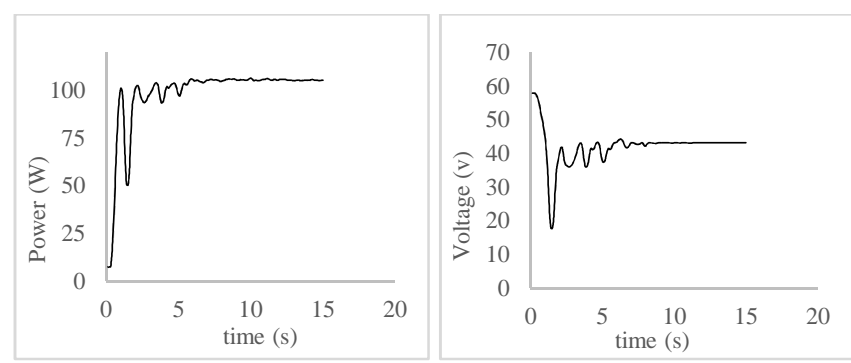

(c)

Fig. 13 Power and Voltage Tracking Curves of Several Methods (a) Conventional PSO; (b) Modified PSO; (c) Proposed AVPSO

Based on Figure 13 (c), the higher oscillation just on the first and second cycle; after that, it will decrease dramatically; the adaptive update particle process causes it. Particle position changes depend on the current position to 
the best position, when the distance is far, then position change will, therefore, be significant. When the current position is close to the best position, the update particle value is small. Therefore, the particle does not move too far from its best position. Another case with conventional PSO and modified PSO, because their update process is constant, the particle update position slowly and gradually changes until it reaches convergent condition.

Modified PSO was displayed to know the effect of changing the value of $\mathrm{C}_{1} \& \mathrm{C}_{2}$. Modified PSO changed the $\mathrm{C}_{1} \& \mathrm{C}_{2}$ value from 1 (standard value PSO) to 0.5 . Based on the experimental result, changing the value of $C_{1} \& C_{2}$ to smaller can cause a lower oscillation level, but it will increase the tracking time because its update position value is smaller. Therefore, AVPSO is proposed to overcome the oscillation level problem without increasing tracking time.

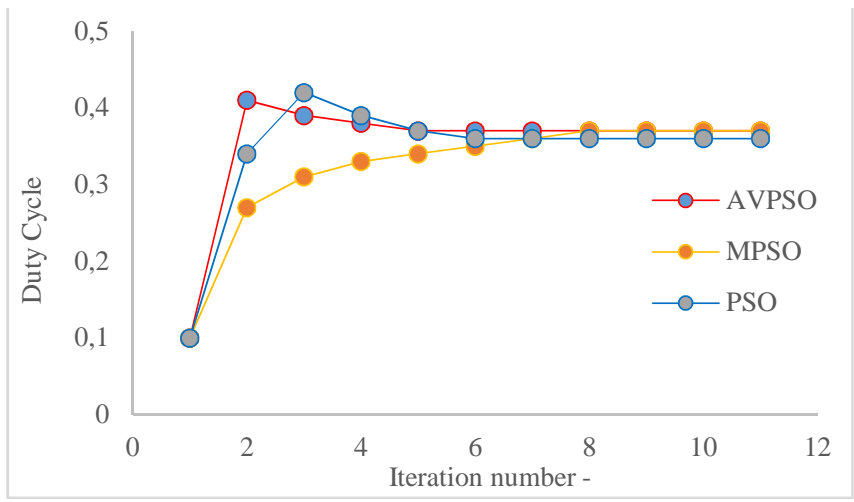

Fig. 14 Movement of Particles in Each Iteration

The duty cycle is an output from each algorithm to control the SEPIC Converter. The duty cycle is representing the particle position of each algorithm. Based on fig 14 , AVPSO's particle moves sharply at the second iteration, and then it decreases gradually until the convergence point. The particle position of PSO still increases until the third iteration, and then it just decreases at the fourth iteration. However, the particle position of MPSO always moves gradually until it reaches the convergent point, it never gets higher than the convergent point, but it has the longest time to reach the convergent point.

3) Modification when there is a change in irradiation or changes in the NUIC pattern: The causes of NUIC are very diverse, thus, the surface pattern that covered is also easy to change. It can change the peak power value and can change the position of the global peak. When there is a change in irradiation or changes in the NUIC pattern, particle positions that were previously at a global peak point may not be at the global peak of the current curve characteristic that was changed. To overcome the problem, it is proposed modification in the algorithm, when the system detects the power to change more than 10 watt after the algorithm reaches the convergent point. The value of power change determined with the aim of getting a sensitive value. The value is not set too small to avoid detection errors when there is no change in irradiation.

To validate the modification, hardware experiments have been carried out. The condition change is made by changing the pattern of PV array surface that covered. The condition changed after the algorithm reaches a convergent point. Figure15 shows the result of the proposed modification. Based on Figure15, the proposed algorithm will re-trace by spreading the particles back until they find the new global peak point.

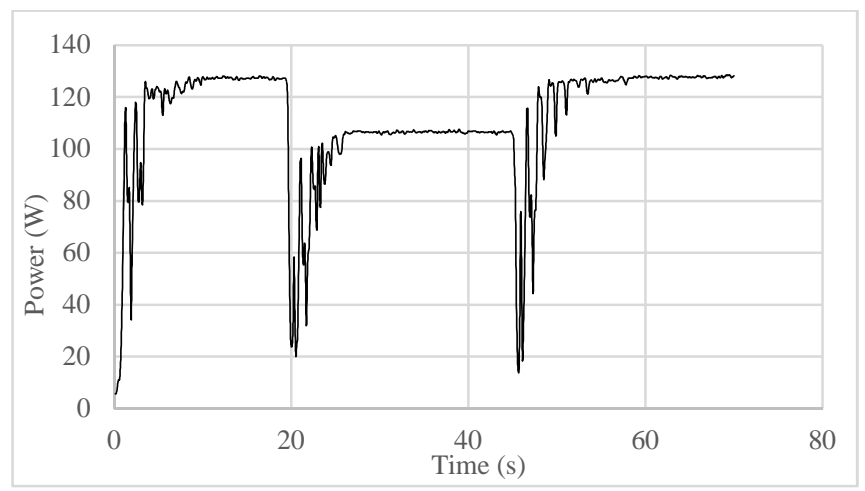

Fig. 15. Power Tracking Curve when There is a Change Power Value

\section{CONCLUSION}

This paper presents the AVPSO (adaptive velocity particle swarm optimization) to track the MPP (maximum power point) of the PV array. The proposed algorithm improves PSO in tracking MPP by modifying the weight factor and acceleration coefficient $\left(\mathrm{C}_{1} \& \mathrm{C}_{2}\right)$ to be an adaptive value. This modification is proposed to overcome the power oscillation level during the tracking period and get tracking time shortly without trapping at a local peak. This paper compared the proposed AVPSO with P\&O to validate that AVPSO is not trapped at a local peak under NUIC. Furthermore, the proposed AVPSO is also compared with conventional PSO to validate its advantages to overcoming power oscillation level and tracking time. Based on several hardware experiments, this fact has been proved through the results provided. The AVPSO algorithm successfully overcomes local peak trapping problem, and also have a low oscillation power level and short tracking time. The proposed AVPSO has an average accuracy of more than $97 \%$ for any condition. This paper also proposes the AVPSO that has been developed in a way so that it is able to work on static and dynamic GMPPT performances.

\section{ACKNOWLEDGMENT}

We would like to thank the Ministry of Education and Culture Republic Indonesia for their support and fund to this research. Also, we would like to extend our gratitude to Politeknik Elektronika Negeri Surabaya for providing us with the access to the laboratorium.

\section{REFERENCES}

[1] P. Nataraj and V. Agarwal, "Distributed PV power extraction based on a modified interleaved SEPIC for nonuniform irradiation conditions," IEEE J. Photovoltaics, vol. 5, no. 5, pp. 1442-1453, Sep. 2015.

[2] N. S. Shah and H. H. Patel, "Evaluating the output power of a PV system under uniform and non-uniform irradiance", in International Conference on Power and Embedded Drive Control (ICPEDC), 2017.

[3] K. Ishaque and Z. Salam, "A deterministic particle swarm optimization maximum power point tracker for photovoltaic system under partial shading condition," IEEE Transactions on Industrial Electronics, vol. 60, Aug. 2013. 
[4] Y. H. Liu and S. C. Huang, "A particle swarm optimization-based maximum power point tracking algorithm for PV systems operating under partially shaded conditions," IEEE Transactions on Energy Conversion, vol. 27, Dec. 2012.

[5] H. S. Sahu and S. K. Nayak,"Extraction of maximum power from a PV array under nonuniform irradiation conditions," IEEE Transactions on Electron Device, vol. 63, Dec. 2016.

[6] K. L. Lian and J. H. Jhang, "Maximum power point tracking method based on perturb-and-observe combined with particle swarm optimization," IEEE J. Photovoltaics,vol. 4, March. 2014.

[7] N. Femia, G. Petrone, G. Spagnuolo, and M. Vitelli, "Optimization of perturb and observe maximum power point tracking method," IEEE Trans. Power Electron, vol. 20, pp. 963-973, Jul. 2005.

[8] R. Faraji, A. Rouholamini, H. R. Naji, R. Fadaeinedjad, and M. R. Chavoshian, "FPGA-based real time incremental conductance maximum power point tracking controller for photovoltaic systems," IET Power Electron, vol. 7, pp. 1294-1304, May. 2014.

[9] T. Sen, N. Pragallapati, and V. Agarwal, "Global maximum power point tracking of PV arrays under partial shading conditions using a modified particle velocity-based PSO technique", IET Renewable Power Generation, vol. 12, pp. 555-564, Dec. 2017.

[10] S. Rajendran and H. Srinivasan, "Simplified accelerated particle swarm optimisation algorithm for efficient maximum power point tracking in partially shaded photovoltaic systems," IET Renewable Power Generation, May. 2016.

[11] Wahjono, E., Anggriawan, D. O., Sunarno, E., Nugraha, S. D., \& Tjahjono, A. (2017). Maximum Power Point Tracking of Photovoltaic Module for Battery Charging Based on Modified Particle Swarm Optimization. In International Review on Modelling and Simulations (IREMOS) (Vol. 10, No. 1, pp. 77-84).
[12] Nugraha, S. D., Wahjono, E., Sunarno, E., Anggriawan, D. O., Prasetyono, E., \& Tjahjono, A. (2016, September). Maximum power point tracking of photovoltaic module for battery charging based on modified firefly algorithm. In 2016 International Electronics Symposium (IES) (pp. 238-243). IEEE.

[13] K. Sundareswaran, S. Peddapati, and S. Palani, "MPPT of PV systems under partial shaded conditions through a colony of flashing fireflies," IEEE Trans. Energy Convers, vol. 29, pp. 463-472, Jun. 2014.

[14] S. Mohanty and B. Subudhi,"A new MPPT design using Grey Wolf optimization technique for photovoltaic system under partial shading conditions," IEEE Tran. Sustainable Energy, vol. 7, Jan. 2016.

[15] Tjahjono, A., Anggriawan, D. O., Habibi, M. N., \& Prasetyono, E. Modified Grey Wolf Optimization for Maximum Power Point Tracking in Photovoltaic System under Partial Shading Conditions.

[16] K. Ishaque and Z. Salam, "An improved particle swarm optimization(PSO)-based MPPT for PV with reduced steady-state oscillation," IEEE Tran. Power Electronics, vol. 27, Aug. 2012.

[17] S. Mohanty, B. Subudhi, and P. K. Ray, "A new MPPT design using grey wolf optimization technique for photovoltaic system under partial shading conditions," IEEE Trans. Sustain. Energy, vol. 7, pp. 181-188, Jan. 2016.

[18] H. Ardi, A. Ajami, and M. Sabahi, "A SEPIC-based high step-up DC-DC converter integrating coupled inductor for renewable energy applications," in Power Electronics, Drive Systems \& Technologies Conference (PEDSTC), April 2017.

[19] O. Kircioğlu, M. Ünlü, and S. Çamur, "Modeling and analysis of DC-DC SEPIC converter with coupled inductors," in International Symposium on Industrial Electronics (INDEL), Dec. 2016. 